\title{
Puzzling Snowballs: Main Belt Comets
}

\author{
Bin Yang and Karen Meech \\ Institute for Astronomy, University of Hawaii, USA \\ email: yangbin@ifa.hawaii.edu
}

\begin{abstract}
Main belt comets (MBCs) are a class of newly discovered objects that exhibit cometlike appearances and yet are dynamically indistinguishable from ordinary main belt asteroids. The measured size and albedo of MBCs are similar to those of classical comets. At present, six MBCs have been discovered, namely 133P/Elst-Pizarro, 176P/LINEAR, 238P/Read, P/2008 R1, P/La Sagra and P/2006 VW139. The total number of active MBCs is estimated to be at the level of a few hundreds (Hsieh \& Jewitt, 2006). Several explanations for the activity of MBCs have been suggested. These include impact ejection, sublimation and rotational instability. However, since renewed activity has been observed in $133 \mathrm{P}$ and $238 \mathrm{P}$ at successive perihelion passages, the most likely explanation may be a thermally-driven process - e.g sublimation of exposed surface ice. Although the proximity of MBCs to the Sun $(\mathrm{r} \sim 3 \mathrm{AU})$ makes the survival of surface ice improbable, thermal models have shown that water ice is thermally stable under a regolith layer a few meters thick. The study of MBCs has recently been complicated by the discoveries of two asteroid collisional events (P/2010 A2 (LINEAR) and (596) Scheila) in 2010, where comet-like dust coma/tail have been attributed to recent impacts. If MBCs are indeed icy, they represent the closest and the third established reservoir of comets (after the Oort cloud and the Kuiper belt). As such, they may have been an important source of water for the Earth's oceans. I will review the current state of MBC studies, present the latest observational results and discuss possible mechanisms that could produce the observed activity. I will also talk about current and future space missions that are dedicated or closely related to MBC studies.
\end{abstract}

